awareness brought about by citizen concern during the 1980 s, led to a dramatic decline in drinking and driving in the industrialized world. Based on various degrees of impairment with special reference to automobiles on public roads, it became imperative for setting maximum, allowable blood alcohol content (BAC) levels as a tool for enforcement and prevention.

The most significant aspect of the BAC value is the legal limit set in each country. While almost all developed countries adhere strictly to the BAC level limits, legislation in transition countries, including Nigeria, does not incorporate legal BAC levels and their implications. Except for South Africa and Zimbabwe, no other African country seems to have any tangible $B A C$ research or legislation.

Methods: A premium digital alcohol Breathalyzer called AlcoScan CA2000 from Craig Medical was utilized to obtain BAC levels from three designated collation centers. The collation centers were the hospital, resting spot, and highways. A computer Excel package was used to analyze the findings.

Results: In the hospital, $73.97 \%$ of males and $26.02 \%$ of females of the total participants were screened with different BAC levels. BAC levels $>0.08 \%$ were found in $43.89 \%$ of drivers or bikers, while $56.11 \%$ had BAC levels between $0.00 \%-0.08 \%$.

Conclusion: The incidence of drinking and driving with specific numeric values of BAC levels was established in this study, thus confirming that several road traffic crashes were alcohol-related. Therefore, there is a need for advoca$c y$, legislation, and sanction of impaired drivers with significant BAC levels in Nigeria.

Keywords: blood alcohol content (BAC); legislation; Nigeria; traffic Prehosp Disast Med 2005;20(2):s78-s79

\section{Epidemiology of Road Traffic Crashes in Ghana from 1993 to the Second Quarter of 2004: Where Do We Go from Here? \\ E. Okparavero \\ Ghana}

Road traffic crashes continue to be a leading cause of death and injuries in West Africa. In Ghana, attempts have been made to curb this disastrous trend, but these efforts have been hampered by the lack of adequate data that could serve as a danger signal to appropriate authorities.

According to police data, from 1994-1998, road traffic crashes were a leading cause of death and injuries in Ghana. However, this dangerous trend seems to be receding, as indicated by data collected from the National Road Safety Commission, the Motor Traffic and Transport Unit (MTTU) of the Ghana police force, and the Building and Road Research Institute (BRRI); all which show a decline in the incidence of road traffic crashes in the years 2000-2003.

Assessment of the importance of trauma relies on accurate sources of data concerning its incidence and outcome, and this study serves to act as a clarion call to the relevant authorities to intervene before the situation becomes even more dangerous. Keywords: Africa; assessment; data; Ghana; injuries; road traffic crashes

Prebosp Disast Med 2005;20(2):s79
Epidemiology of Traffic Crashes at Various Ages in Rafsanjan, Iran

H. Bakbshi

Rafsanjan Medical University, Iran

Introduction: Traffic crashes are major medical problems. They are the largest factor for mortality and morbidity among individuals $<45$ year of age.

Objective: To understand the epidemiology of traffic crashes in order to prevent them.

Methods: A descriptive study of 936 persons injured in traffic crashes was carried out at the Ali-Ebn Abitaleb Emergency Center in Rafsanjan, Iran, over a 122-day period (366 shifts), in every season of the year 2000 in a selected stratified randomly method.

Results: A total of $82 \%$ of traffic crash victims were male, $73.29 \%$ were $<30$ years of age, and $34.63 \%$ were single. The mean age was $24.6 \pm 16$ years in males and $25 \pm 16.4$ years in females. A total of $33.17 \%$ of the crashes occurred in the city of Rafsanjan, $33.07 \%$ were in rural areas, and $54.35 \%$ of the crashes occurred around and outside the city. A total of $40.35 \%$ of the traffic crashes occurred in the morning. Damage to extremities (46\%), head injuries (27.24\%), and multiple fractures $(16.55 \%)$ were the most prevalent type of body injuries. A total of $94.5 \%$ of patients were treated at out-patient clinics, $15.87 \%$ were hospitalized, and the morbidity rate was $1.5 \%$.

Conclusions: High rates of traffic injuries for people $<40$ years old demands education of motor vehicle laws and regulations with the consideration of safety points in order to prevent crashes. Preventive measures should be evaluated. Keywords: Iran, Rafsanjan, safety; traffic crashes

Prebosp Disast Med 2005;20(2):s79

\section{Patterns of Facial Bone Fractures following Road} Traffic Crashes: A Benin City Experience

O. Osaiyuzu

University of Benin, Nigeria

The frequencies of fractures of the various facial bones vary from region to region. This is due partly to the most common etiologic factor obtaining in such a region. The commonest etiologic factor in this environment is road traffic crashes (RTCs). Others are gunshots, fights, falls, and industrial accidents.

This prospective study was conducted to determine the pattern of facial bone fractures following road traffic crashes in Benin City, Nigeria. During a six-month period, 89 patients with facial bone fractures as a result of road traffic crashes were studied.

Results showed that more males than females were involved, with the majority between the ages of 21-30 years. Minibuses (35\%) and motorcycles (29\%) were responsible for more accidents than other vehicular types. Mandibular fractures were found to be the most common $(41.6 \%)$, and therefore the fracture most likely to confront the dental surgeon.

Keywords: Benin City; facial bones; fracture; Nigeria; truffic crashes Prehosp Disast Med 2005;20(2):s79 